\title{
Focus on radiation exposure from cardiovascular imaging with computed tomography
}

\author{
Paul Schoenhagen $\cdot$ Hans Reiber
}

Received: 11 February 2009/Accepted: 11 February 2009/Published online: 4 March 2009

(C) Springer Science+Business Media, B.V. 2009

After the recently published results from the PROTECTION trial [1], which demonstrate large differences in radiation exposure for cardiovascular indicted CT scans between experienced centers, in this issue Gopal et al. [2] describe potential significant reduction of radiation exposure using prospective triggering and lower tube voltage in selected patients. The editors would also like to announce an upcoming Focus Issue of the journal dedicated to various dose saving strategies. Guest-edited by Frank Rybicki, $\mathrm{MD}, \mathrm{PhD}$ of Brigham and Women's in Boston, a series of articles from a group of international experts, will discuss the impact of state-of-the-art scanner technology of all major CT manufactures.

Since its introduction to diagnostic medical imaging in the 1970s [3], the use of computed tomography has risen sharply and has allowed more precise diagnosis of a wide range of conditions, including cardiovascular disease. However, the increased use is

Editorial comment on 9396 (DOI: 10.1007/s10554-008-9396-z.)

P. Schoenhagen $(\square)$

Imaging Institute and Heart \& Vascular Institute,

Cleveland Clinic, Desk J-1 4, 9500 Euclid Avenue,

Cleveland, OH 44195, USA

e-mail: schoenp1@ccf.org

H. Reiber

Department of Radiology, Division of Image Processing, Leiden University Medical Center, Leiden,

The Netherlands associated with rising radiation exposure [4], and recent articles have highlighted its potential longterm side effects [5]. The general principle in medicine that the diagnostic or therapeutic intervention should not be worse than the disease, is reflected by the 'ALARA principle (as low as reasonably achievable)' in diagnostic imaging using ionizing radiation [6].

In order to minimize the radiation exposure from CT in general and for the individual patient in particular, it is important to consider appropriate clinical indications and scan protocols. Appropriateness criteria for cardiovascular $\mathrm{CT}$ have been published [7]. In the realm of vascular imaging, CT is typically used initially instead of another tests associated with exposure to ionizing radiation. If, as would be the case for a patient with suspected pulmonary embolism or aortic dissection, CT provides a reliable diagnosis and makes nuclear imaging or angiography unnecessary, CT is considered appropriate. On the other hand, if a positive coronary CTA would most likely need confirmation by conventional angiography, as would be likely in a patient with known CAD or high pre-test probability, the test is not considered appropriate. It is also important to consider, that the relatively non-invasive nature of a test alone does not establish its usefulness for screening (or supports performance out of curiosity), in particular because false-positive test in patients with low pre-test probability can be associated with untoward outcome [8]. 
Once the appropriate indication of CT is established for a specific patient, it is critical to choose the most appropriate protocol using state-of-the art equipment. However, there is a wide variety of different protocols and a lack of standardization. This is confounded by rapid technical developments in the field of cardiovascular CT in the past few years [9, 10]. A central challenge in cardiovascular CT is the rapid motion of the heart and subsequent need to synchronize data acquired during several heartbeats to the identical cardiac phase in the RR-interval. Modern scanners with fast gantry rotation times and large number of detectors allow two general approaches. In helical acquisitions, data is acquired throughout the cardiac cycle but subsequent reconstructions use only data from limited, typically diastolic, windows ('spiral imaging with retrospective gating'). These protocols allow review of multiple reconstructions along the cardiac cycle in case of motion artifact and also for limited functional assessment. However, because X-ray exposure occurs throughout the cardiac cycle (despite reduced tube current outside a pre-specified, typically diastolic window $=$ 'tube current modulation') these protocols are associated with higher radiation exposure. More recently, sequential acquisition has become possible for cardiovascular CT, where data is acquired only in the pre-specified window of the cardiac cycle ('axial imaging with prospective triggering') [11]. Because the X-ray tube is turned off outside the pre-specified window, radiation exposure is significantly reduced. However, the reduced dose comes at the price of less flexibility for additional reconstructions. Other recent developments include improved use of radiation filters, fast helical scanning, and use of lower tube current voltage $(100 \mathrm{kv})[9,10]$.

The choice between these different protocols is not well standardized, and the PROTECTION trial demonstrate that there are large differences in radiation exposure for cardiovascular indicted CT scans even in experienced centers [1]. In this cross-sectional, international, multicenter, observational study the estimated median radiation dose (dose-length product, DLP) in 1965 patients was $885 \mathrm{mGy} \times \mathrm{cm}$ (IR 568-1259 $\mathrm{mGy} \times \mathrm{cm}$ ), which corresponds to an estimated radiation dose of $12 \mathrm{mSv}$. There was a high variability in median DLP values between study sites, ranging from 331 to $2146 \mathrm{mGy} \times \mathrm{cm}$. In linear regression analysis several dose saving strategies were independently associated with reduced radiation dose, including reduced scan length $(5 \%$; $95 \%$ CI, 4$6 \%$ ), electrocardiographically controlled tube current modulation $(-25 \%$; $95 \% \mathrm{CI},-23$ to $-28 \%$; applied in $73 \%$ of patients), $100-\mathrm{kV}$ tube voltage $(-46 \%$; $95 \% \mathrm{CI},-42$ to $-51 \%$; applied in $5 \%$ of patients), sequential scanning $(-78 \%$; $95 \% \mathrm{CI},-77$ to $-79 \%$; applied in $6 \%$ of patients), and type of 64-slice CT system (for highest vs. lowest dose system, 97\%; 95\% CI, 88-106\%). Importantly, the algorithms for dose reduction were not associated with deteriorated diagnostic image quality in this observational study.

The article by Gopal et al. [2] in this issue describe a retrospective analysis of CT scans performed in 149 consecutive patients. The authors compare prospective and retrospective protocols and the additional use of $100 \mathrm{kV}$ scanning using 64-MDCT scanners. Two different voltages were utilized based on body habitus ( $100 \mathrm{kVp}$ for a weight $<85 \mathrm{~kg}$, or standard $120 \mathrm{kVp}$ for a weight $>85 \mathrm{~kg}$ ). Radiation doses and image quality (signal to noise ratio) was measured for each patient, and compared between different acquisition protocols. Use of $100 \mathrm{kVp}$ reduced radiation dose by $41.5 \%$ using prospective triggering and $39.6 \%$ using retrospective imaging as compared to $120 \mathrm{kVp}(P<0.001)$. Use of prospective imaging reduced radiation exposure by $82.6 \%$ as compared to retrospective imaging $(P<$ 0.001). Using both prospective imaging and $100 \mathrm{kV}$ radiation dose was reduced by $90 \%(P<0.001)$. The prospective ECG-triggered acquisition and $100 \mathrm{kVp}$ images were of diagnostic quality, allowing adequate assessment in all patients.

The reduction of exposure associated with prospective imaging is larger than reported in other recent publications and the results may be limited by selection bias and small subgroups, but the trend is consistent with the literature. In particular, the absolute dose of $1.9 \mathrm{mSv}$ for coronary CTA with prospective scanning at $100 \mathrm{kV}$ appears achievable [11]. As pointed out by Achenbach in an accompanying editorial the goals of high diagnostic image quality and low radiation dose need to be balanced in individual patients, "even if it requires a little extra effort".

We hope that both the article by Gopal et al., and the upcoming Focus Issue will contribute to the discussion about appropriate use of computed tomography in cardiovascular imaging. 


\section{References}

1. Hausleiter J, Meyer T, Hermann F, Hadamitzky M, Krebs M, Gerber TC, McCollough C, Martinoff S, Kastrati A, Schomig A, Achenbach S (2009) Estimated radiation dose associated with cardiac CT angiography. JAMA 301:500507

2. Gopal A, Mao SS, Karlsberg D, Young E, Waggoner J, Ahmadi N, Pal RS, Leal J, Karlsberg RP, Budoff MJ (2008) Radiation reduction with prospective ECG-triggering acquisition using 64-multidetector computed tomographic angiography. Int J Cardiovasc Imaging. doi:10.1007/ s10554-008-9396-Z

3. Hounsfield GN (1980) Computed medical imaging. Science 210:22-28

4. Brenner DJ, Hall EJ (2007) Computed tomography-an increasing source of radiation exposure. N Engl J Med 357:2277-2284

5. Einstein AJ, Henzlova MJ, Rajagopalan S (2007) Estimating risk of cancer associated with radiation exposure from 64-slice computed tomography coronary angiography. JAMA 298:317-323

6. Gerber TC, Carr JJ, Arai AE, Dixon RL, Ferrari VA, Gomes AS, Heller GV, McCollough CH, McNitt-Gray MF, Mettler FA, Mieres JH, Morin RL, Yester MV (2009) Ionizing radiation in cardiac imaging: a science advisory from the American Heart Association Committee on Cardiac Imaging of the Council on Clinical Cardiology and Committee on Cardiovascular Imaging and Intervention of the Council on Cardiovascular Radiology and Intervention. Circulation 119:1056-1065

7. Hendel RC, Patel MR, Kramer CM, Poon M, Hendel RC, Carr JC, Gerstad NA, Gillam LD, Hodgson JM, Kim RJ, Kramer CM, Lesser JR, Martin ET, Messer JV, Redberg RF, Rubin GD, Rumsfeld JS, Taylor AJ, Weigold WG, Woodard PK, Brindis RG, Hendel RC, Douglas PS, Peterson ED, Wolk MJ, Allen JM, Patel MR (2006) ACCF/ ACR/SCCT/SCMR/ASNC/NASCI/SCAI/SIR 2006 appropriateness criteria for cardiac computed tomography and cardiac magnetic resonance imaging. J Am Coll Cardiol 48:1475-1497

8. Nissen SE (2008) Limitations of computed tomography coronary angiography. J Am Coll Cardiol 52:2145-2147

9. Petersilka M, Bruder H, Krauss B, Stierstorfer K, Flohr TG (2008) Technical principles of dual source CT. Eur J Radiol 68:362-368

10. Rybicki FJ, Otero HJ, Steigner ML, Vorobiof G, Nallamshetty L, Mitsouras D, Ersoy H, Mather RT, Judy PF, Cai T, Coyner K, Schultz K, Whitmore AG, Di Carli MF (2008) Initial evaluation of coronary images from 320detector row computed tomography. Int $\mathrm{J}$ Cardiovasc Imaging 24:535-546

11. Husmann L, Valenta I, Gaemperli O, Adda O, Treyer V, Wyss CA, Veit-Haibach P, Tatsugami F, von Schulthess GK, Kaufmann PA (2008) Feasibility of low-dose coronary CT angiography: first experience with prospective ECGgating. Eur Heart J 29:191-197 